John A. Kellum Dilhari R. DeAlmeida Valerie J. Watzlaf

\title{
eResearch: the case of acute kidney injury
}

Received: 30 October 2012

Accepted: 31 October 2012

Published online: 7 December 2012

(C) Springer-Verlag Berlin Heidelberg and ESICM 2012

This editorial refers to the article available at:

doi: 10.1007/s00134-012-2767-x.

\section{J. A. Kellum · D. R. DeAlmeida}

Center for Assistance in Research using eRecord (CARe),

University of Pittsburgh, Pittsburgh, PA, USA

\section{J. A. Kellum (楩)}

Department of Critical Care Medicine, The Clinical Research, Investigation, and Systems Modeling of Acute Illness (CRISMA) Center, School of Medicine, University of Pittsburgh, Room 604 Scaife Hall, 3550 Terrace Street, Pittsburgh, PA 15261, USA e-mail: kellumja@ccm.upmc.edu

Tel.: 412-647-6966

Fax: 412-647-8060

D. R. DeAlmeida · V. J. Watzlaf

Department of Health Information Management,

School of Health and Rehabilitation Sciences,

University of Pittsburgh, Pittsburgh, PA, USA

This year, an estimated 2 million people will die of acute kidney injury (AKI) worldwide [1]. Acute kidney injury is a serious complication of acute illness, typically occurring as a result of a combination of exposures like sepsis or nephrotoxins, and susceptibilities such as advanced age and underlying chronic kidney disease (CKD). Acute kidney injury has been shown to be independently associated with death and worsening of existing, or development of new, CKD [2]. The incidence of AKI and how it impacted survival was not known until recently. The reason was that AKI (or acute renal failure as it was known then) was a clinical diagnosis without a clear definition. While the condition was easier to recognize when it was severe, there was uncertainty as to whether early or milder forms of renal dysfunction were significant. However, in 2004, the acute dialysis quality initiative (ADQI) proposed new consensus criteria for diagnosing and staging AKI [3]. These criteria included changes in serum creatinine and urine output as the basis for diagnosis and staging and used the acronym RIFLE for three levels of severity (risk, injury and failure) and two outcomes (loss and end-stage kidney disease). These criteria have been subsequently adopted with minor modifications by the acute kidney injury network [4], and the modified RIFLE criteria have been endorsed by the kidney disease improving global outcomes (KDIGO) clinical practice guideline on AKI [5]. Indeed, this guideline attaches specific actions to the stages of AKI based on these criteria. These criteria for AKI have now been validated in thousands of patients [6], and their development has helped create a common epidemiological framework of reference. Furthermore, these criteria have increased the clinical appreciation that even small increases $(>25 \mu \mathrm{mol} / \mathrm{L})$ in serum creatinine are associated with greater risk of death and helped provide a structure for clinical trials both in terms of enrollment criteria and endpoints.

The RIFLE criteria are easy to apply at the bedside and, therefore, have direct clinical application. However, serum creatinine and to a lesser extent, the urine output, are also contained in the modern electronic medical record (EMR). A definition for AKI that is easily mapped to the EMR has enabled much of the rapid acquisition of new epidemiologic data for this condition over the past few years. Indeed, we have seen an unprecedented increase in information concerning the attack rates, risk factors, treatments and both short and long term outcomes for AKI in just the last eight years. Much of this growth can be attributed to the ease of which AKI can be extracted from the EMR. 
Thus, in a very real way, AKI is a prime topic for a new science known as "eResearch". eResearch can be defined as the use of information technology (IT) and information management (IM) technologies to conduct research in a more meaningful way [7]. eResearch enables the development of novel high performance computing technologies such as predictive modeling and/or dynamic systems modeling [8]. eResearch is a tool for personalized or "precision" medicine and AKI is "ready" for precision medicine [9]. Prevention of AKI in individuals may involve a precise balance of fluid and vasopressors to ensure renal perfusion, but avoid fluid overload. Timing of initiation and discontinuation and, indeed, even selection of who should receive renal replacement therapy (RRT) are open questions. Once the decision to start RRT has been made, intensity has only been established for the "average" patient and precise matching of intensity to patient needs in the context of residual renal function and recovery is far beyond current practice.

Finally, AKI contributes to medication failure or toxicity because of an inability to properly dose, for example, essential drugs like antibiotics in the setting of unstable elimination rates and volumes of distribution. The role for precision medicine guided by eResearch in addressing these needs is enormous. The stakes are very high. The independent association between AKI and mortality is strong and long lasting: the increased risk of death or end stage renal disease can be measured for at least 1 year following the index event [10].
Perhaps the next step in the evolution of precision medicine for AKI has been taken by Mandelbaum and colleagues [11] in this issue. The authors analyzed very detailed EMR data from more than 17,000 patients and examined various combinations of creatinine/urine output thresholds and observation periods and generated contour plots for relationships to in-hospital mortality and prevision of RRT. Not surprisingly, changes in creatinine and urine output both showed an interaction with time. The longer the abnormality the worse the outcome, although large absolute changes in creatinine were less time dependent. Of course, when changes are short-lived, it usually means recovery and recovery usually correlates with better outcome.

The good news from the analysis by Mandelbaum and colleagues [11] is that their results strongly support the current KDIGO recommendations based on the modified RIFLE criteria [12]. The better news perhaps is that the contour plots may be used to provide a more precise measure of risk because they may vary by institution or patient type. Such analyses could thus transform how we diagnose and/or stage AKI by developing more personalized metrics rather than relying on a one-size-fits-all approach. Furthermore, by adding additional dimensions, such as the results of novel biomarkers for AKI, these analyses may greatly improve our diagnostic and prognostic ability and bring us that much closer to the realm of precision medicine.

\section{References}

1. Ali T, Khan I, Simpson W, Prescott G, Townend J, Smith W, Macleod A (2007) Incidence and outcomes in acute kidney injury: a comprehensive population-based study. J Am Soc Nephrol 18:1292-1298

2. Chawla LS, Amdur RL, Amodeo S, Kimmel PL, Palant CE (2011) The severity of acute kidney injury predicts progression to chronic kidney disease. Kidney Int 79:1361-1369

3. Bellomo R, Ronco C, Kellum JA, Mehta RL, Palevsky P, The ADQI workgroup (2004) Acute renal failuredefinition, outcome measures, animal models, fluid therapy and information technology needs: the second international consensus conference of the ADQI Group. Crit Care 8:R204R212

4. Mehta RL, Kellum JA, Shah SV, The Acute Kidney Injury Network (2007) Acute kidney injury network: report of an initiative to improve outcomes in acute kidney injury. Crit Care 11:R31
5. Kidney Disease: Improving Global Outcomes (KDIGO) (2012) Clinical practice guideline for acute kidney injury. Kidney Int Suppl 2:89-115

6. Ricci Z, Cruz D, Ronco C (2008) The RIFLE criteria and mortality in acute kidney injury: a systematic review. Kidney Int 73:538-546

7. http://www.monash.edu.au/eresearch/ (Reviewed on 10/26/2012)

8. Solovyev A, Mikheev M, Zhou L, Dutta-Moscato J, Ziraldo C, An G, Vodovotz Y, Mi Q (2010) SPARK: a framework for multi-scale agent-based biomedical modeling. IJATS 2:18-30

9. Matheny M, Miller R, Alp Ikizler T, Waitman L, Denny J, Schildcrout J, Dittus R, Peterson J (2010)

Development of inpatient risk stratification models of acute kidney injury for use in electronic health records. Med Decis Making 30:639 originally published online 30 March 2010 doi: 10.1177/0272989X10364246
10. Murugan R, Karajala-Subramanyam V, Lee M, Yende S, Kong L, Carter M, Angus DC, Kellum JA, Genetic and Inflammatory Markers of Sepsis (GenIMS) Investigators (2010) Acute kidney injury in non-severe pneumonia is associated with an increased immune response and lower survival. Kidney Int 77:527-535

11. Mandelbaum T, Lee J, Scott DJ, Mark RG, Malhotra A, Howell MD, Talmor D (2012) Empirical relationships among oliguria, creatinine, mortality, and renal replacement therapy in the critically ill. Intensive Care Med. doi: 10.1007/s00134-012-2767-x

12. Kidney Disease: Improving Global Outcomes (KDIGO) Acute Kidney Injury Work Group (2012) KDIGO clinical practice guideline for acute kidney injury. Kidney inter 2(Suppl): 1-138 